\title{
Antropología
}

\section{Metaestudio cualitativo sobre vivencias y gestión del cotidiano en adultos mayores que padecen enfermedades crónicas}

\section{Qualitative meta-study on daily experiences and management in older adults with chronic diseases}

\section{Meta-estudo qualitativo sobre as experiências e gestão diárias em idosos com doenças crônicas}

Sandra Milena Hernández Zambrano ${ }^{1}$, César Hueso Montoro² ${ }^{2}$ Rafael Montoya Juárez ${ }^{3}$, José Luis Gómez Urquiza ${ }^{4}$, Candela Bonill de las Nieves. ${ }^{5}$

${ }^{1}$ Enfermera, PhD Student, Universidad de Alicante, España. Responsable de Formación Continuada, Fundación Index, Granada. ${ }^{2}$ Enfermero, PhD. Facultad de Ciencias de la Salud, Universidad de Granada.

${ }^{3}$ Enfermero, PhD. Facultad de Ciencias de la Salud, Universidad de Granada.

${ }^{4}$ Enfermero, PhD Student, Universidad de Granada, España. Facultad de Ciencias de la Salud, Universidad de Granada.

${ }^{5}$ Enfermera PhD. Unidad de Digestivo y Endoscopias. Hospital Torrecárdenas. Almería.

Cómo citar este artículo en edición digital: Hernández Zambrano, S.M., Hueso Montoro, C., Montoya Juárez, R., Gómez Urquiza, J.L. y Bonill de las Nieves, C. (2016). Metaestudio cualitativo sobre vivencias y gestión del cotidiano en adultos mayores que padecen enfermedades crónicas. Cultura de los Cuidados (Edición digital), 20, 44. Disponible en: < http://dx.doi.org/10.14198/cuid.2016.44.07>

Correspondencia: C/ San Jerónimo, n5, 5B CP:18001 Granada, España. Teléfono / fax: (34)958-293304

Correo electrónico: samyta599@hotmail.com

Recibido: 08/08//2015; Aceptado: 09/11/2015

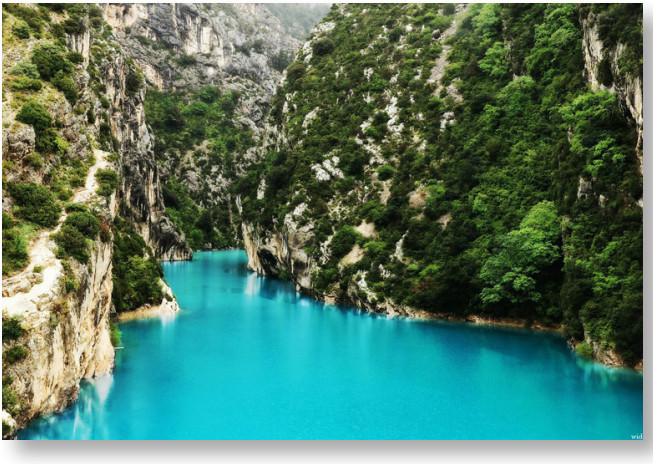

ABSTRACT

Aims: To describe the cultural, interpersonal and personal experience of older adults with chronic diseases and to identify the strategies and support networks used by them in their daily environment for managing the disease.

Methods: A qualitative meta-analitic study was developed in four stages: literature search, studies categorization, methodological quality assessment and results analysis.

Results: A sample of 22 qualitative studies was obtained. The findings were organized into 4 categories: Compression of the condition, daily self-management, perception of health services and the caregiver's daily life.

Conclusions: The effort of older adults to achieve a new social and personal balance is articulated in self-management strategies that they use to face their sufferings. Interrelationships with health services, family and support networks are essential for chronicity management.

Key words: Aged, Chronic disease, Qualitative Research, Activities of daily living, Self care 


\section{RESUMO}

Objetivo: Descreva a experiência cultural, interpessoal e pessoal dos idosos com doenças crônicas e identificar estratégias e redes de apoio que eles usam em seu ambiente todos os dias para controlar a doença.

Método: Um tipo qualitativo meta-meta foi desenvolvido. A estratégia metodológica consistiu em quatro etapas: pesquisa bibliográfica, categorização dos estudos, avaliação da qualidade metodológica e análise dos resultados.

Resultados: Uma amostra bibliográfica de 22 estudos qualitativos foi obtido. Os resultados foram organizados em quatro categorias: condição de compressão, a auto-gestão no cotidiano, Percepção dos Serviços de Saúde e cuidador Daily.

Conclusões: $\mathrm{O}$ esforço de adultos mais velhos para alcançar um novo equilíbrio social e pessoal é dividido em estratégias de auto-gestão que eles usam no seu dia a cumprir as suas condições. Inter-relações com os serviços de saúde, redes de apoio à família são essenciais para a gestão crônica.

Palavras-chave: Idoso, Doença crônica, Pesquisa qualitativa, Atividades da vida diária, Autocuidado.

\section{RESUMEN}

Objetivo: Describir la experiencia cultural, interpersonal y personal de los adultos mayores con enfermedades crónicas e identificar las estrategias y redes de apoyo que utilizan en su entorno cotidiano para gestionar la enfermedad.

Método: Se desarrolló un metaestudio tipo metaanálisis cualitativo. La estrategia metodológica constó de cuatro etapas: Búsqueda bibliográfica, Categorización de los estudios, Evaluación de la calidad metodológica y Análisis de resultados.
Resultados: Se obtuvo una muestra bibliográfica de 22 estudios de naturaleza cualitativa. Los hallazgos se organizaron en 4 categorías: Compresión del padecimiento, Autogestión en el cotidiano, Percepción de los Servicios de Salud y Cotidiano del cuidador.

Conclusiones: El esfuerzo de los adultos mayores por alcanzar un nuevo equilibrio social y personal se articula en las estrategias de autogestión que utilizan en su día a día para afrontar sus padecimientos. Las interrelaciones con los servicios de salud, familia y redes de apoyo son fundamentales para gestionar la cronicidad.

Palabras clave: Anciano, Enfermedad crónica, Investigación cualitativa, Actividades cotidianas, Autocuidado.

\section{INTRODUCCIÓN}

La tendencia del envejecimiento de la población asociada con el aumento de las enfermedades crónicas se ha convertido en un reto importante para la salud pública. La creciente prevalencia de enfermedades crónicas degenerativas representa un alto costo económico y social para los individuos y sus familias.

El incremento en la esperanza de vida es un fenómeno que se viene evidenciando en la población mundial. En 2050, el mundo contará con 2000 millones de personas mayores de 60 años, de las cuales cerca de un $85 \%$ vivirá en países en desarrollo (OMS, 2008). El envejecimiento y los nuevos modos de vida a nivel mundial están haciendo que las enfermedades crónicas no transmisibles sean una causa cada vez más importante de morbilidad y mortalidad. En el 2008, de los 57 millones de defunciones que se produjeron en el mundo, 36 millones se debieron a Enfermedades no Transmisibles (ENT), principalmente en- 
fermedades cardiovasculares, cáncer, diabetes y enfermedades pulmonares crónicas (OMS, 2011).

Responsables gubernamentales y los diferentes actores de los sistemas de salud, han comenzado a reconocer la necesidad de un cambio en el modelo de asistencia sanitaria para afrontar el reto que plantea la transición epidemiológica (Jadad et al., 2010; Nuno-Solinís et al., 2013; Corrales-Nevado el al., 2012). El modelo más popular a nivel global, es el propuesto por la OMS denominado The Innovative Care for Chronic Conditions framework (ICCC) [Perfil de la Atención Innovadora para las condiciones crónicas (AICC)] (OMS, 2002) que a nivel micro representa la interacción entre los profesionales de los servicios de salud, el paciente y la comunidad. El calificativo activado para el paciente se transforma en motivado y preparado (Jadad et al., 2010; OMS, 2002).

Al igual que la transición epidemiológica exige nuevos modelos de asistencia sanitaria y de formación profesional, la investigación en ciencias de la salud, requiere nuevas propuestas de investigación que exploren las enfermedades crónicas desde la perspectiva de los individuos y sus familias. Amezcua (2010), a través del enfoque de la Investigación Aplicada a los Cuidados (IAC) propone reflexionar sobre un tipo de investigación centrada más en la persona que en la enfermedad. Este modelo sugiere la Investigación sobre el Cotidiano del Sujeto (ICS) como un área donde se construye el conocimiento (Amezcua, 2010; Amezcua \& Hernández Zambrano, 2012).

La ICS está ubicada en el paradigma constructivista y ámbito teórico de la microsociología, que se ocupa del comportamiento social cotidiano y defiende que el conocimiento sobre el mundo cotidiano que los profesionales de la salud comparten con el paciente y su fa- milia (la salud, la enfermedad, el padecimiento, la necesidad, el cuerpo, la familia, la relación terapéutica, el cuidado, la cultura), es una fuente de conocimientos útiles para la toma de decisiones en el cuidado de la salud (Amezcua \& Hernández Zambrano, 2012).

Estudios como los de Thorne et al. (2012), Paterson (2001), Rogers (2009) y MercadoMartínez \& Hernández-Ibarra (2007), proponen recurrir a la investigación sobre las enfermedades crónicas desde una perspectiva constructivista para explorar la dimensión subjetiva del padecimiento. En las últimas dos décadas, se ha producido una rápida proliferación de investigaciones cualitativas sobre la naturaleza de vivir con una enfermedad crónica. Tanto en lo social como en las ciencias de la salud, diversas cuestiones teóricas, clínicas y prácticas han llevado a los investigadores a comprender la enfermedad crónica y proponer marcos teóricos que puedan facilitar la atención de estos padecimientos (Ingadottir \& Halldorsdottir, 2008)

Este incremento de estudios cualitativos sugiere la necesidad de desarrollar trabajos de carácter secundario que permitan evaluar críticamente la literatura publicada al respecto e integrar los hallazgos de los mejores estudios existentes. Por ello, el objetivo de esta investigación fue desarrollar un metaestudio cualitativo para describir la experiencia cultural, interpersonal y personal de los adultos mayores con enfermedades crónicas e identificar las estrategias y redes de apoyo que utilizan en su entorno cotidiano para gestionar la enfermedad.

\section{MÉTODO}

Se desarrolló un Metaestudio tipo metaanálisis cualitativo. Se trata de diseño de investigación secundaria caracterizado por el reanálisis de varios estudios cualitativos, por tanto, se 
aplica una técnica de análisis cualitativo a datos de diferentes estudios previamente identificados mediante un complejo método de revisión sistemática que implica el análisis crítico de los mismos (Gálvez Toro, 2003).

La estrategia metodológica constó de cuatro etapas: Búsqueda bibliográfica, Categorización de los estudios, Evaluación de la calidad metodológica y Análisis de resultados. La pregunta que orientó la búsqueda fue: ¿Cómo es la vivencia de los adultos mayores que padecen enfermedades crónicas y qué estrategias y recursos utilizan en su entorno cotidiano para gestionar la enfermedad?

\section{Estrategia de búsqueda.}

Se realizó una búsqueda en las bases de datos MEDLINE, LILACS, SCIELO, IBECS, CUIDEN, y La Biblioteca Cochrane. Los términos seleccionados (Anciano, Anciano de 80 o más años, Enfermedad Crónica, Investigación cualitativa, Actividades Cotidianas, enfermedades cardiovasculares, diabetes mellitus, neoplasias, hipertensión, Enfermedades respiratorias) se adecuaron a los descriptores MESH, DECs y tesauro de CUIDEN, lo que nos permitió emplearlos en inglés, portugués y español.

\section{Criterios de inclusión y exclusión.}

Se incluyeron estudios cualitativos primarios (originales) y secundarios (revisiones sistemáticas cualitativas, metaanálisis cualitativos, metasintesis cualitativas), con enfoque descriptivo e interpretativo. La búsqueda se limitó a estudios publicados en el periodo de 2001 a 2011 en tres idiomas: portugués, español e inglés.

Se incluyeron estudios cuya población fueron sujetos mayores de 60 años, diagnosticados con una o más de las siguientes enfermedades crónicas: diabetes, cáncer, enfermedades respiratorias crónicas, enfermedades cardiovasculares e hipertensión. Según la OMS estas enfermedades son las principales causas de mortalidad en el mundo, siendo responsables del 63\% de las muertes (OMS, 2008).

Se han incluido 4 dimensiones de análisis que corresponden a las grandes categorías de la ICS, a) Comprensión del padecimiento ante la enfermedad crónica; b) Cultura del cuidado; c) Percepción sobre los servicios de salud; d) El cotidiano del Cuidador.

\section{Proceso de selección de los estudios.}

Una vez ejecutadas las ecuaciones de búsqueda, se identificaron 3927 títulos y resúmenes. Para refinar la búsqueda se filtraron las Bases de Datos por idioma, periodo de publicación y tipo de estudio dando como resultado 472 registros. Se procedió a revisar el título y resumen de los registros encontrados para verificar el tema de investigación y diseño metodológico y establecer un primer filtro de selección. En esta fase se eliminaron los registros duplicados y aquellos estudios que no cumplían con los criterios de inclusión.

En esta segunda fase, se revisó el texto completo de los artículos potencialmente seleccionados como elegibles, evaluándose las características de la población y la coherencia del objetivo y los hallazgos con las dimensiones que se planteaban explorar en el metaestudio. La muestra bibliográfica provisional estuvo constituida por 23 artículos.

La revisión crítica de la calidad metodológica de cada estudio se realizó utilizando como referencia la guía CASPE de lectura crítica de estudios cualitativos y la guía CASPE de lectura crítica de Revisiones sistemáticas (Cano Arana, González Gil \& Cabello López, 2010). Con el objetivo de evaluar tanto la calidad me- 
todológica como la utilidad de los hallazgos en la práctica clínica se ha incluido en la plantilla la clasificación de los hallazgos del estudio según diseño y nivel de utilidad, propuesta por Gálvez Toro (2003). Se excluyó uno de los estudios que no cumplía con los criterios de rigor metodológico dando como resultado una muestra de 22 estudios. (Ver Gráfica 1. Diagrama de flujo)

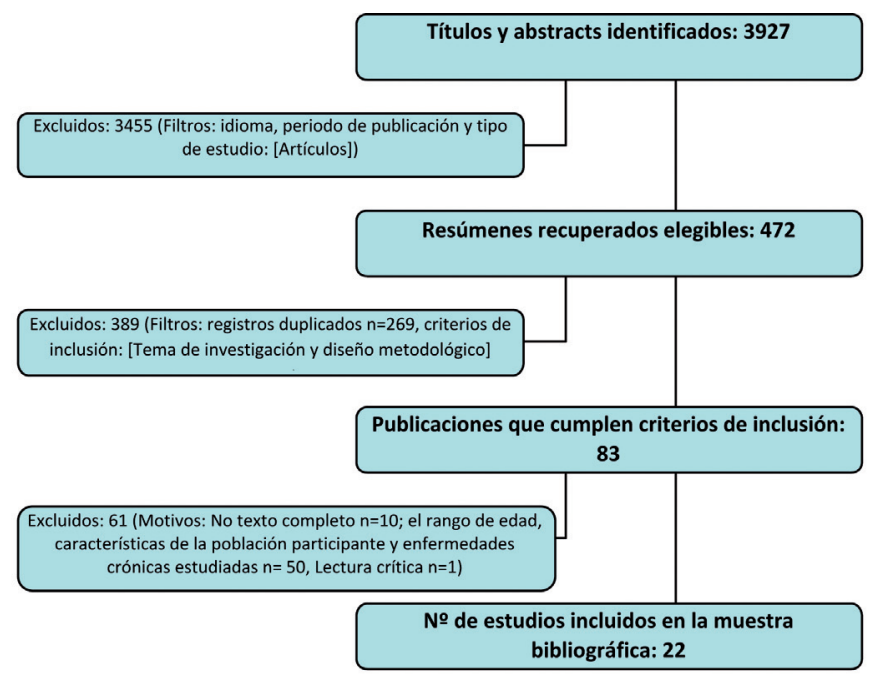

Gráfico 1. Diagrama de Flujo

Fuente: Elaboración propia cación axial permitió relacionar los hallazgos de los 22 estudios, generando un análisis integrado de resultados. Los códigos sustantivos se organizaron por afinidades encontrando patrones en conjunto para construir categorías. Para garantizar el rigor metodológico se realizó triangulación de los hallazgos entre investigadores.

\section{RESULTADOS}

\section{Caracterización de los estudios}

Para la descripción de los hallazgos se diferenciaron y etiquetaron los 22 estudios incluidos en la muestra bibliográfica con código compuesto por la letra "E” seguida de un número. En la tabla 1 describimos, el código de cada estudio, referencia bibliográfica, diseño metodológico, número de informantes incluidos y su país de procedencia.

La producción científica se concentró en los EE.UU con un $35,5 \%$, seguida de Suecia con un $17,7 \%$ y de Brasil con un 13,3 \%.

\section{Análisis y categorización de resultados.}

Se creó una base de datos Excel que nos permitió categorizar los hallazgos de cada estudio a partir de las 4 categorías que nos propusimos explorar. Tras una lectura detallada y minuciosa de los resultados y las conclusiones de cada estudio se extrajeron y clasificaron los hallazgos a partir de las características definidas en cada categoría.

Para realizar un análisis más exhaustivo de cada categoría, se siguió la propuesta analítica de Strauus y Corbin (2002) buscando, mediante la codificación abierta y axial, subcategorías emergentes que permitieran una mejor comprensión del fenómeno. La codifi-
En consonancia con estos datos, el 63,6\% de la producción científica esta publicada en inglés, el $27,2 \%$ en portugués y el $9 \%$ en español.

El total de sujetos que incluían los 22 estudios seleccionados asciende a 1032 personas mayores con enfermedades crónicas. En la mitad de los estudios el rango de edad osciló entre los 60 a 85 años y en los estudios restantes, las poblaciones de estudio contenían una importante cohorte de adultos mayores, tal que la edad media de los participantes correspondió a 60 años o más.

Los padecimientos crónicos en el cotidiano de los adultos mayores son explicados a partir de cuatro categorías: Comprensión del padeci- 
miento, percepción de los servicios de salud, el cotidiano de los cuidadores y autogestión en el cotidiano.

\section{Comprensión del padecimiento}

Las huellas de los signos y síntomas físicos de la enfermedad es una sub-categoría que nos describe la enfermedad desde su dimensión biológica avalada por los signos y síntomas físicos que comprometen el bienestar de los ancianos y se convierten en la primera manifestación consciente de la enfermedad (E6, E11, E12 E15, E19 y E22). Gullick y Stainton (E12) afirman que la experiencia de no tener aire suficiente para la eficacia y la movilidad se encuentran en el primer plano de la conciencia para las personas con EPOC (E12).

La metamorfosis del cuerpo es una experiencia corporeizada de la enfermedad, producida por los cambios en los cuerpos atribuidos a la enfermedad, efectos del tratamiento y cambios relacionados con la edad o con las co-morbilidades (E11, E12 y E15,) Visentin y Lenardt (E6) en su estudio sobre el cáncer, afirman que para las personas las enfermedades que no son visibles, no presentan un importante grado de compromiso.

\section{Las cargas emocionales.}

Los adultos mayores describen una amplia gama de emociones negativas causadas por sus padecimientos: incertidumbre, miedo, ansiedad, inseguridad, actitudes de enfrentamiento y rechazo de sí mismo, cambios en el temperamento, incluyendo la ira, irritabilidad, frustración, beligerancia y terquedad, tristeza, desesperación, deseos de llorar, irritabilidad, depresión y su poca tolerancia ante situaciones adversas. Las personas mayores podrían sentirse gravemente preocupadas y ansiosas al confrontarse con su enfermedad (E5, E12, E15 y E22).
Frente a las profundas cargas emocionales, el sentimiento de esperanza se convierte en un aliciente para sobrellevar la enfermedad (E6, E11, E15 y E18). Las garantías están firmemente ancladas en lo sagrado, la familia, profesionales y personas cercanas a él. La esperanza es compartida por las oraciones de los vecinos, amigos y la comunidad a la que pertenece (E6) y se refleja en su actitud ante la vida diaria: "Agarrar la vida con las dos manos, jestamos aquí!. Puede ser que tengamos una enfermedad crónica, que podría ser mayor, pero no vamos a dejar que nos venza" (verbatim E18).

\section{Desintegración de la vida diaria.}

Los adultos mayores describen cómo su vida se desintegra: están totalmente ocupados con los viajes para someterse a exámenes y procedimientos; se reside fuera de casa durante la duración del tratamiento y a continuación se debe hacer frente a los efectos secundarios no sólo en el cuerpo, sino en la vida social y en las relaciones (E8). La enfermedad aparece como una amenaza para sí mismo y para sus relaciones sociales, lo que representa un factor limitante, que dificulta la carga emocional que el paciente debe soportar. Se identifican diferentes pérdidas ocasionadas por los padecimientos crónicos: limitaciones físicas y funcionales que generan temores frente a la dependencia, pérdida de las relaciones sociales con temor al aislamiento, pérdida de la capacidad productiva y pérdidas financieras.

\section{Autogestión en el cotidiano}

Explorar los recursos que utilizan los adultos mayores para gestionar sus padecimientos, tuvo dos objetivos fundamentales: por un lado conocer los conocimientos, creencias y valores culturales que influyen en la toma de decisiones en salud; por otro lado, describir su trayec- 
toria de cuidado: identificación de su situación de salud, toma de decisiones y ejecución de prácticas para el mantenimiento, protección y recuperación. Esta categoría sitúa el padecimiento en el contexto social y cultural del paciente, es decir en la dimensión simbólicosocial o sickness.

\section{Saberes populares.}

Lo hallazgos reflejan la medicina alternativa y tradicional como saberes complementarios más no excluyentes. Visentin y Lenardt (E6) refieren cómo la medicina tradicional popular deja de ser un conjunto fragmentado de las prácticas de curación, para convertirse en un sistema complejo y articulado de conocimientos sobre la vida, enfermedad y muerte.

Una de la prácticas más generalizadas de los saberes populares se produce a través de grupos religiosos, la oración, curanderos y sanadores que utilizan rituales y tradiciones. Las personas utilizan la sabiduría popular para sugerir el uso de métodos terapéuticos (E6). ${ }^{19}$ La resistencia a los sufrimientos, la confianza en Dios y la fe trae comodidad, seguridad, paz, tranquilidad, serenidad y agradecimiento, tiene una influencia decisiva en las enfermedades porque les ayuda a sobrellevarlas o las cura (E15 y E22). Desde esta perspectiva, la fe es el reflejo de lo que implica una actitud distinta frente a la vida y no puede seguir siendo vista como una actitud anticientífica, sino que debiera ser reconocida como una expresión de subjetividades que contribuye al cuidado, protección y restauración de la salud.

\section{Gestión del autocuidado.}

Las estrategias de autogestión fueron identificadas en 14 de los estudios incluidos. Actitudes de auto-empoderamiento que incluyen sentimientos de responsabilidad de la propia salud; la conciencia del poder personal y la fuerza de haber sufrido un duro período de la enfermedad y tratamiento; la capacidad y el deseo de aprender; la utilización de experiencias previas para gestionar su enfermedad; las prácticas de autocuidado para modificar estilos de vida; la evaluación de sus condiciones físicas y funcionales para la toma de decisiones y la autonomía física cognitiva y financiera, garantiza a los adultos mayores una actitud activa hacia la vida para hacer frente a su enfermedad.

\section{Percepción sobre los sistemas de salud}

Esta categoría exploró como perciben los enfermos crónicos a los actores y recursos implicados en el sistema de salud: las instituciones, profesionales, tratamientos e intervenciones.

\section{Factores a nivel macro y meso que condicio-} nan las intervenciones en salud dirigidas a los adultos mayores.

Algunos estudios describen las barreras que perciben los adultos mayores e interfieren con la gestión de sus enfermedades. Por un lado, influye la situación de desamparo de las políticas de protección al anciano y a esta situación se suma la inequidad de acceso a los servicios de salud y a las tecnologías disponibles, que son fundamentales en el proceso de coordinación asistencial para el manejo de las enfermedades crónicas (E1, E4 y E13).

\section{Percepciones sobre la relación con los profe- sionales sanitarios.}

A nivel micro, la percepción sobre la interrelación con los profesionales de salud tiene dos matices que interfieren en la toma de decisiones frente al manejo de la enfermedad. Por un lado, se refleja un modelo paternalista y autoritario caracterizado por una atención desinteresada, que impone, genera dependen- 
cia e invisibiliza al adulto mayor en la toma de decisiones sobre su salud. Por otro lado, se identifica un modelo horizontal basado en la confianza, que fomenta la participación y la implicación de la persona en la gestión de su enfermedad, mejorando así la eficacia en los resultados de salud.

Una relación terapéutica fundamentada en un modelo horizontal, basado en la confianza con los profesionales, que fomenta la participación e implica a los adultos mayores en la toma de decisiones, se asocia con una mejora de la atención, incluyendo un mejor reconocimiento de los problemas y adherencia al tratamiento.

\section{Percepción sobre los tratamientos farmacoló- gicos}

Las limitaciones físicas y funcionales interfieren en la adherencia al tratamiento ya que, tal y como muestran algunos estudios, la discapacidad visual y cognitiva impone limitaciones para los pacientes (E7, E8 y E14). La función cognitiva tiende a disminuir con el factor edad, lo que ofrece dificultades para recordar los horarios, las dosis y la ingesta de los medicamentos (E7). Acordarse de tomar los medicamentos fue uno de los problemas más comunes reportados por los participantes de un estudio (E14).

No obstante, y a pesar de sus limitaciones, los adultos mayores movilizan recursos que favorecen la continuidad de su tratamiento terapéutico. La autogestión en los tratamientos farmacológicos implica la participación activa de los pacientes en el proceso de toma de su medicación (E7, E8, E11, E13, E14 y E18).

\section{Información y comunicación: desaciertos y oportunidades.}

La información y la comunicación en la relación terapéutica es una pieza clave para los adultos mayores a la hora de tomar decisiones sobre su cuidado. Algunos estudios describen los desaciertos y oportunidades de los profesionales sanitarios en su relación terapéutica con los pacientes crónicos (E8, E10, E14, E15, E21). Como desaciertos refieren que se ha disminuido la confianza y la capacidad para comunicarse eficazmente con sus proveedores de atención en salud; las diferencias lingüísticas y el nivel de alfabetización que no son tenidos en cuenta por los profesionales (E10) la falta de información que conlleva a las percepciones erróneas y las acciones consiguientes de los participantes en relación a su tratamiento; la búsqueda de otras fuentes de información tales como libros, folletos y programas de televisión cuando los profesionales sanitarios no son una fuente de información directa y adecuada a sus preocupaciones o a su situación y la falta de disposición de los profesionales para proporcionar información individualizada $\mathrm{y}$ específica (E14).

En cuanto a las oportunidades, se argumenta que la información se debe dar desde el momento del diagnóstico y durante todo el transcurso del tratamiento y debe ser repetida debido a las dificultades de los adultos mayores para recordar. Los beneficios que provee un relación terapéutica fundamentada en la comunicación garantiza que la información sobre los medicamentos fortalezca las percepciones de los participantes sobre su eficacia percibida, o que los pacientes puedan monitorizar la progresión de su enfermedad y/o entender cómo funciona su medicamento (E14).

\section{El cotidiano de los cuidadores}

La familia, los cuidadores informales y las redes sociales desempeñan un papel importante en el mantenimiento, protección y recuperación de la salud de los adultos mayores con 
enfermedades crónicas. Esta categoría refleja dos matices en las relaciones familiares que influyen en las emociones y toma de decisiones de los adultos mayores. Por un lado las relaciones familiares otorgan nuevos significados a la enfermedad trasformando el padecimiento en una experiencia positiva que favorece la autogestión. No obstante, los adultos mayores también relatan dificultades en las relaciones familiares lo que contribuye al desarrollo o el empeoramiento de su enfermedad.

Cuando las relaciones familiares están fundamentadas en la cooperación y co-responsabilidad para la toma de decisiones sobre el itinerario terapéutico (evaluación de alternativas, movilización de recursos o fortalecimiento de las relaciones conyugales) provén un recurso esencial para los adultos mayores en la gestión de las enfermedades crónicas. Por el contrario, la desintegración de la vida familiar derivada de las cargas físicas, emocionales, sociales y económicas de la enfermedad, genera dificultades en las relaciones familiares que impactan negativamente en el bienestar de las personas. Desde la perspectiva de los adultos mayores, las redes informales constituyen un importante recurso para la autogestión de su enfermedad ya que les ofrece apoyo, compañía en el sufrimiento, les dan sentido de pertenencia en su comunidad y potencia la socialización además de proporcionar asesoramiento y material de apoyo. La familia, los miembros de la iglesia, los vecinos, clubes de enfermos y vecinos constituyen una red de apoyo fundamental (E2, E15, E16 y E22).

\section{DISCUSIÓN}

El padecimiento en primera persona hace referencia a la experiencia cultural, interpersonal y personal de la enfermedad (Hueso, 2006). Investigadores como Peterson (2001),

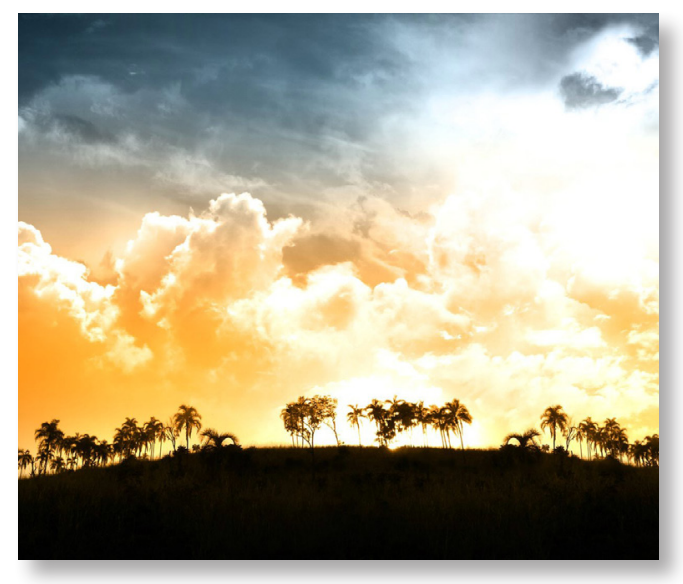

Amezcua (2000), Siles (2003) y Hueso et al. (2012), reconocen la importancia de comprender los aspectos inherentes a la subjetividad de la enfermedad desde la perspectiva de los sujetos. Indagar sobre los padecimientos crónicos en el cotidiano de los adultos mayores, nos permitió explorar la complejidad de la enfermedad desde una triple dimensión: el plano de las molestias físicas o sintomáticas (disease), el plano de las interpretaciones o significados de la persona que lo está viviendo (illness) y las interpretaciones y significados asociados a la enfermedad que son vividos como una experiencia socialmente constituida (sickness) (Hueso, 2006).

Los hallazgos reflejan, como los síntomas se convierten para los adultos mayores en indicadores objetivos de la patología. Paterson (2001) identifica que cuando las personas se concentran en los síntomas, les ayuda a proveer pruebas para que otras personas reiteren que su enfermedad es verdadera. Esta visión puede ser el resultado del modelo positivista que ha primado sobre la comprensión de la enfermedad desde un punto de vista biologicista, que solo prioriza la alteración orgánica, obviando la subjetividad inherente al proceso salud-enfermedad (Amezcua, 2000; Rodríguez, 2011). 
En contraposición a esta visión reduccionista, han emergido modelos para el abordaje de la cronicidad como el denominado "Cambio de perspectivas" ("The shifting perspectives") que argumenta la complejidad de vivir con enfermedades crónicas y la describe como un proceso en curso y continuamente cambiante sobre la enfermedad, lo que permite que las personas den sentido a su experiencia (Paterson, 2001). Los resultados del metaestudio corroboran la importancia de la dimensión subjetiva de la enfermedad que responde a cómo se siente el sujeto, cuáles son sus vivencias y cómo afecta a su vida. Cuando a un paciente se le diagnostica una enfermedad crónica, implica la interrupción de las actividades cotidianas, readaptación al entorno social y la redefinición del papel que representan los individuos en la sociedad (Rogers, 2009.) Para los adultos mayores, padecer enfermedades crónicas no es un proceso lineal, simple o predecible. Hay un cúmulo de experiencias culturales y de condiciones sociales e históricas que otorgan un sentido de complejidad y dialécticas a su enfermedad y que deben confrontar en su día a día.

Aunque en la literatura científica se ha evidenciado la influencia positiva de la comunicación sobre el cumplimiento terapéutico y la satisfacción del usuario (Almendro-Padilla et al., 2013; Danet et al., 2012) los hallazgos del metaestudio reflejan la supremacía de un modelo de relación profesionales-pacientes paternalista y vertical que invisibiliza al adulto mayor en la toma de decisiones sobre su salud.

Recientemente, los cambios sociales ponen en relieve la importancia de la comunicación como requisito previo a la autonomía del paciente y a su derecho a la información (Barca Fernández et al., 2004). Se propone potenciar un modelo de relación más deliberativo y participativo, entre unos profesionales sanitarios menos protagonistas y unos pacientes más expertos e informados (Barca Fernández et al., 2004; Wagner Todd et al., 2001).

Según Ojeda et al. (2006), la confianza depositada en el profesional es un elemento central. Hay evidencia de que cuando los pacientes pueden expresar sus puntos de vista, problemas y preocupaciones, y se toman decisiones consensuadas, es más probable que se impliquen y asuman mayor responsabilidad en el cuidado de su propia salud 46-49 (Ojeda et al. 2006; Barry et al. 2000).

Los modelos más destacados de gestión de las enfermedades crónicas ["Modelo de Atención a Enfermedades Crónicas" ("Chronic Care Model"), y "Modelo de Atención Innovadora a Condiciones Crónicas" ("Innovative Care for Chronic Conditions Framework")], ratifican la importancia de situar a los pacientes informados, motivados y preparados como elemento central de un sistema que cuenta con un equipo proactivo de profesionales con los conocimientos y experiencia necesarios (Bengoa et al. 2010). Este cambio de enfoque ha supuesto la emergencia de diferentes iniciativas innovadoras orientadas a la autogestión de las enfermedades crónicas, toma de decisiones compartidas, promoción del autocuidado, coaching de salud, entre otras (McGowan et al. 2010; Bonal et al. 2012).

Por ejemplo, el programa emblemático del Reino Unido "Paciente Experto" (The Expert Patient), convierte al propio paciente en protagonista de su salud. Considera como puntos clave en el tratamiento de las enfermedades crónicas el autocuidado y autotratamiento, el tratamiento de la enfermedad, la gestión de casos y gestión del autoconocimiento (Romina Pérez, 2010). El paciente aumenta la comprensión sobre su condición y mejora su calidad de vida. (Sans Corrales et al. 2012) 
Los resultados del metaestudio reafirman la necesidad de este cambio de enfoque orientado hacia un marco de relación entre pacientes y profesionales más horizontal y dialógico. Las diferentes estrategias de autogestión identificadas en el estudio, desvelan como las actitudes y acciones de empoderamiento de los pacientes, les garantizan una mayor implicación y participación en la toma de decisiones para el cuidado de su salud. Paterson 11 en su modelo explicativo de las enfermedades crónicas, expresa que los pacientes aprenden cuánto poder tiene ellos sobre su enfermedad, crean ambientes alentadores, desarrollan destrezas personales y comparten sus conocimientos de la enfermedad con otros.

\section{Limitaciones}

Dos cuestiones metodológicas deben ser consideradas. En primer lugar que el proceso de búsqueda se hizo exclusivamente en bases de datos electrónicas lo que influye en el tamaño de la muestra bibliográfica. El seguimiento de la literatura gris, la búsqueda manual de revistas y la consulta de expertos ampliaría la muestra del metaestudio.

En segundo lugar, aunque todos los trabajos incluidos son de naturaleza cualitativa y representan las experiencias de los adultos mayores con enfermedades crónicas, la muestra es heterogénea por la diversidad de contextos, enfoques de investigación y perspectivas teóricas de los estudios incluidos.

\section{CONCLUSIONES}

Los principales hallazgos se puedan expresar en un conjunto de implicaciones prácticas que conlleva a los profesionales sanitarios a brindar una atención más cercana a la realidad de los adultos mayores: Comprender los padecimientos crónicos más allá de su dimensión biológica; evaluar con detalle el entorno cotidiano de los adultos mayores; promover la autogestión de las enfermedades crónicas; desarrollar competencias culturales que favorezcan el reconocimiento de los saberes populares como complementarios y no excluyentes de la medicina tradicional; promover modelos de relación profesionales-pacientes basados en la confianza; evaluar el impacto de las relaciones familiares e identificar y valorar las redes de apoyo que utilizan los adultos mayores para gestionar su enfermedad.

El esfuerzo de los adultos mayores por alcanzar un nuevo equilibrio social y personal se articula en las estrategias de autogestión que utilizan en su día a día para afrontar sus padecimientos. En este sentido, las interrelaciones con los servicios de salud, familia y redes de apoyo son fundamentales para gestionar la cronicidad.

Los alcances de la investigación nos dirigen a continuar con futuras investigaciones sobre el cotidiano de los adultos mayores con padecimientos crónicos. Las investigaciones focalizadas en el cotidiano de los pacientes y sus familias aportan evidencias útiles, pertinentes y contextuales para la toma de decisiones en el cuidado de la salud.

\section{BIBLIOGRAFÍA}

- Almendro-Padilla, C., García-Vicente, S., VázquezCosta, M. y Blanes-Pérez, M.C. (2013). El riesgo y su comunicación a los pacientes para la toma de decisiones en salud. SEMERGEN. Medicina de Familia, 39(7), 386-390.

- Amezcua, M. (2000). Enfermedad y padecimiento: significados del enfermar para la práctica de los cuidados. Cultura de los Cuidados, 7-8, 60-67.

- Amezcua, M. (2010). Investigación Aplicada en Cuidados de Salud. Index de Enfermería, 19(4), 237-239.

- Amezcua, M. y Hernández Zambrano, S.M. (2012). Investigación sobre el cotidiano del sujeto: oportunidades para una ciencia aplicada. Texto Contexto Enferm, 21(3), 675-683.

- Barca Fernández, I., Parejo Miguez, R., Gutiérrez Martín, P., Fernández Alarcón, F., Alejandre Lázaro, G. y López de Castro, F. (2004). La información al paciente y su participación en la toma de decisiones clínicas. Aten Primaria, 33(7), 361-7. 
- Barry, C.A., Bradley, C.P., Britten, N., Stevenson, F.A. y Barber N. (2000). Patients' unvoiced agendas in general practice consultations: qualitative study. BMJ, 320, 1246-50.

- Bengoa, R., Martos, F., Nuño, R., Kreindler, S., Novak, T., y Pinilla, R. [Contribuyentes principales] Management models. En: Jadad, A.R., Cabrera, A., Martos, F., Smith, R. y Lyons R.F. (2010). When people live with multiple chronic diseases: a collaborative approach to an emerging global challenge. Granada: Escuela Andaluza de Salud Pública.

- Bonal Ruiz, R., Almenares Camps, H.B., y Marzán Delis, M. (2012). Coaching de salud: un nuevo enfoque en el empoderamiento del paciente con enfermedades crónicas no transmisibles. MEDISAN, 16(5), 773-785.

- Cano Arana, A., González Gil, T., y Cabello López, J.B. Plantilla para ayudarte a entender un estudio cualitativo. En: CASPe. Guías CASPe de Lectura Crítica de la Literatura Médica. (2010). Cuaderno III (pp. 3-8). Alicante: CASPe.

- Corrales-Nevado, D., Alonso-Babarro, A., y RodríguezLozano, M.A. (2012). Continuidad de cuidados, innovación y redefinición de papeles profesionales en la atención a pacientes crónicos y terminales. Informe SESPAS 2012. Gac Sanit, 26(S), 63-68.

- Danet, A., Escudero, M.J., Prieto, M.A., y March Cerdá, J.C. (2012). Comunicación paciente-médico y toma de decisiones en salud en Andalucía. Expectativas de pacientes crónicos del SSPA. Revista de Comunicación y Salud, 2(2), 63-77.

- Gálvez Toro, A. (2003). Clasificación de las Evidencias por su Diseño y Utilidad. La investigación secundaria cualitativa. Index Enferm, (43), 45-49.

- Gestión para la salud [Sede web] . 2010. [Consultado el junio 8 de 2014]. De Romina Pérez, D. Análisis sistémico de la gestión e intervención de las enfermedades crónicas en los sistemas sanitarios. Recuperado de: http://easpgps. wordpress.com/2010/03/10/analisis-sistemico1-de-lagestion-e-intervencion-de-las-enfermedades-cronicasen-los-sistemas-sanitarios/

- Hueso, C. (2006). El padecimiento ante la enfermedad. Un enfoque desde la teoría de la representación social. Index Enferm, 15(55), 49-53.

- Hueso, C., Siles, J., Amezcua, M., Bonill de las Nieves, C., Pastor Montero, S., y Celdrán Mañas, M. (2012). Comprendiendo el padecimiento humano ante la enfermedad: manifestaciones, contexto y estratégias . Rev. Latino-Am. Enfermagem, 20(3), 619-628.

- Ingadottir, B. y Halldorsdottir, S. (2008). To discipline a "dog": the essential structure of mastering diabetes. Qual Health Res, 18(5), 606-19.

- Jadad, A.R., Cabrera, A., Martos, F., Smith, R., y Lyons R.F. (2010). When people live with multiple chronic diseases: a collaborative approach to an emerging global challenge. Granada: Escuela Andaluza de Salud Pública.

- McGowan, P., Lorig, K., Jadad, AR., Armayones, M., Bender, J., Gil-Zorzo, E. et al. [Contribuyentes principales] Educación de los pacientes y apoyo a la autogestión. En: Jadad, A.R., Cabrera, A., Martos, F., Smith, R., y
Lyons R.F. (2010). When people live with multiple chronic diseases: a collaborative approach to an emerging global challenge. Granada: Escuela Andaluza de Salud Pública.

- Mercado-Martínez, F.J. y Hernández-Ibarra, E. (2007). Las enfermedades crónicas desde la mirada de los enfermos y los profesionales de la salud: un estudio cualitativo en México. Cad Saúde Pública, 23(9), 2178-2186.

- Nuno-Solinís, R., Fernández-Cano, P., Mira-Solves, J., Toro-Polanco, N., Contel, J., Guilabert-Mora, M. et al. (2013). Desarrollo de IEMAC, un Instrumento para la Evaluación de Modelos de Atención ante la Cronicidad. Gac Sanit, 27(2), 128-134.

- Ojeda Feo, J., Freire Campo, J.M., y Gérvas Camacho, J. (2006). La coordinación entre Atención Primaria y Especializada: ¿reforma del sistema sanitario o reforma del ejercicio profesional? Rev Adm Sanit, 4(2), 357-82.

- Organización Mundial de La Salud (2008). Informe sobre la salud en el mundo 2008. La Atención Primaria en Salud más necesario que nunca. Informe de un Grupo Científico OMS. Ginebra: OMS.

- Organización Mundial de la Salud (2011). Informe sobre la situación mundial de las enfermedades no transmisibles 2010. Resumen de orientación. Informe de un Grupo Científico de la OMS. Ginebra: OMS.

- Paterson, B. (2001). The shifting perspectives model of chronic illness. Journal of Nursing Scholarship, 33(1), 21-6.

- Rogers, A. (2009). Consideraciones sobre la autogestión de las enfermedades Crónicas. Index Enferm, 18(4), 253257.

- Rodríguez, J.M. y Soto, E.C. (2011). Determinación y causalidad en salud colectiva: algunas consideraciones en torno a sus fundamentos epistemológicos. Ciênc. saúde coletiva, 16, (suppl.1), 847-854.

- Sans Corrales, M., Gardeñes Morón, L., Moliner Molins, C., Campama Tutusaus, I., Pérez García, S. y Rozas Martínez, M. (2012). Health care pathways and expert patients: Do they improve outcomes? Int J Integr Care, 12(Suppl2), e31.

- Siles, J. (2003). Antropología y Enfermería. La necesaria simbiosis entre dos disciplinas para vertebrar culturalmente la teoría y la praxis de los cuidados. Index Enferm, 43, 28-32.

- Strauss, A. y Corbin, J. (2002). Bases de la investigación cualitativa. Técnicas y procedimientos para desarrollar la teoría fundamentada. $1^{\text {a }}$ Edición. Medellín: Editorial Universidad de Antioquia.

- Thorne, S., Paterson, B., Acorn, S., Canam, C., Joachim, G. y Jillings, C. (2002). Chronic Illness Experience: Insights from a Metastudy. Qual Health Res, 12, 437.

-Wagner Todd, H., Teh-Wei, H. y Hibbard Judith, H. (2001). The demand for consumer health information. $J$ Health Econom, 20, 1059-75.

-World Health Organization (2002). Innovative care for chronic conditions: building blocks for action: global report. Ginebra: WHO. 
Tabla 1. Caracterización de la muestra bibliográfica

\begin{tabular}{|c|c|c|}
\hline $\begin{array}{c}\text { Código y Referencia } \\
\text { bibliográfica }\end{array}$ & Diseño & $\begin{array}{l}\text { No de informantes y } \\
\text { país de procedencia }\end{array}$ \\
\hline $\begin{array}{l}\text { (E1) Meirelles BHS, Simon CAE, } \\
\text { Vieira FMA, Cortezi MDV, Nativi- } \\
\text { dade MSL. Condições associadas à } \\
\text { qualidade de vida dos idosos com } \\
\text { doença crônica. Cogitare Enferm. } \\
2010 \text { Jul/Set; 15(3): 433-40 }\end{array}$ & Estudio descriptivo & $\begin{array}{c}22 \text { personas. Florianópolis. } \\
\text { Brasil }\end{array}$ \\
\hline $\begin{array}{l}\text { (E2) Ribeiro JP, Rocha SA, Popim } \\
\text { RC. Compreendendo o significado } \\
\text { de qualidade de vida segundo ido- } \\
\text { sos portadores de diabetes mellitus } \\
\text { tipo II. Esc Anna Nery (impr.)2010; } \\
\qquad 14(4): 765-771\end{array}$ & Estudio fenomenológico & 12 personas. Botucatu, Brasil \\
\hline $\begin{array}{l}\text { (E3) Pilger C, Rampari EM, Waid- } \\
\text { man MAP, Carreira L. Hemodiálise: } \\
\text { seu significado e impacto para a } \\
\text { vida do idoso. Esc. Anna Nery. } \\
\text { 2010; 14(4). }\end{array}$ & Enfoque exploratorio cualitativo & 22 personas. Paraná, Brasil \\
\hline $\begin{array}{l}\text { (E4) Sanches MJ, Santana NHS, } \\
\text { Moracvick YAD. Percepção de } \\
\text { idosos hipertensos sobre suas neces- } \\
\text { sidades de saúde. Rev. esc. enferm. } \\
\text { 2012; 46(1). }\end{array}$ & Enfoque cualitativo descriptivo & 32 personas. Marilia, Brasil \\
\hline $\begin{array}{l}\text { (E5) Tenahua Quit I, Díaz Pinzón, } \\
\text { AD, Alonso Castillo M, Medina } \\
\text { López OM. Significado de la ex- } \\
\text { periencia del adulto mayor al vivir } \\
\text { con hipertensión. Desarrollo Científ } \\
\text { Enferm -Méx- | 2006; 14(8):292-296 }\end{array}$ & Enfoque cualitativo descriptivo & 10 personas. Puebla, México \\
\hline $\begin{array}{l}\text { (E6). Visentin A, Lenardt MH. O } \\
\text { itinerário terapêutico: história oral } \\
\text { de idosos com câncer. Acta Paul } \\
\text { Enferm -Bra- .2010; 23(4):486-492. }\end{array}$ & Historia oral & 5 personas. Paraná, Brasil \\
\hline
\end{tabular}




\begin{tabular}{|c|c|c|}
\hline $\begin{array}{l}\text { (E7) Lenardt MH, Hammerschmidt } \\
\text { KSA, Borghi ÂCS, Vaccari É, Seima } \\
\text { MD. O idoso portador de nefropatia } \\
\text { diabética e o cuidado de si. Texto Con- } \\
\text { texto Enferm, 2008; 17(2):313-320 }\end{array}$ & $\begin{array}{l}\text { Estudio de naturaleza cuali- } \\
\text { tativa, de tipo convergente- } \\
\text { asistencial }\end{array}$ & 7 personas. Curitiba, Brasil \\
\hline $\begin{array}{l}\text { (E8) Chen CH, Wu JR, Yen M, Chen } \\
\text { ZC. A model of medication-taking } \\
\text { behavior in elderly individuals with } \\
\text { chronic disease. J Cardiovasc Nurs. } \\
\text { 2007; 22(5): 359-65. }\end{array}$ & Teoría fundada & 19 personas. Tainan, Taiwán \\
\hline $\begin{array}{l}\text { (E9) Helsel D, Mochel M, Bauer } \\
\text { R. Chronic illness and Hmong } \\
\text { shamans. J Transcult Nurs. 2005; } \\
\text { 16(2): 150-4. }\end{array}$ & Estudio exploratorio & 11 personas. California, EE.UU \\
\hline $\begin{array}{l}\text { (E10) Wang J, Matthews JT. Chronic } \\
\text { disease self-management: views } \\
\text { among older adults of Chinese des- } \\
\text { cent. Geriatr Nurs. 2010; 31(2): 86-94. }\end{array}$ & Estudio descriptivo & $\begin{array}{l}19 \text { personas. Pittsburgh } \\
\text { (Pennsylvania) EE.UU }\end{array}$ \\
\hline $\begin{array}{l}\text { (E11) Yu DS, Lee DT, Kwong AN, } \\
\text { Thompson DR, Woo J. Living with } \\
\text { chronic heart failure: a review of } \\
\text { qualitative studies of older people. J } \\
\text { Adv Nurs.2008; 61(5): 474-83. }\end{array}$ & Revisión sistemática cualitativa & $\begin{array}{l}269 \text { personas. EE.UU. } \\
\text { La revisión incluyó } 14 \text { artículos. } \\
\begin{array}{c}(\mathrm{n}=8), \text { Suecia }(\mathrm{n}=5) \text { y } \\
\text { Nueva Zelanda }(\mathrm{n}=1)\end{array}\end{array}$ \\
\hline $\begin{array}{l}\text { (E12) Gullick J, Stainton MC. Living } \\
\text { with chronic obstructive pulmo- } \\
\text { nary disease: developing conscious } \\
\text { body management in a shrinking } \\
\text { life-world. J Adv Nurs. 2008; 64(6): } \\
605-14\end{array}$ & Estudio fenomenológico & $\begin{array}{c}29 \text { personas. New Wales del } \\
\text { Sur, Australia }\end{array}$ \\
\hline $\begin{array}{l}\text { (E13) Jeon YH, Essue B, Jan S, } \\
\text { Wells R, Whitworth JA. Economic } \\
\text { hardship associated with managing } \\
\text { chronic illness: a qualitative inquiry. } \\
\text { BMC Health Serv Res.2009; 9: } 182 .\end{array}$ & Estudio descriptivo & 52 personas. Sydney, Australia \\
\hline
\end{tabular}




\begin{tabular}{|c|c|c|}
\hline $\begin{array}{l}\text { (E14) Gordon K, Smith F, Dhillon S. } \\
\text { Effective chronic disease mana- } \\
\text { gement: patients' perspectives } \\
\text { on medication-related problems. } \\
\text { Patient Educ Couns. 2007; 65(3): } \\
\quad 407-15 .\end{array}$ & Estudio descriptivo & $\begin{array}{c}98 \text { personas. Lambeth, } \\
\text { Southwark y Lewisham (Lon- } \\
\text { dres), Inglaterra }\end{array}$ \\
\hline $\begin{array}{l}\text { (E15) Hughes N, Closs SJ, Clark D. } \\
\text { Experiencing cancer in old age: a } \\
\text { qualitative systematic review. Qual } \\
\text { Health Res. 2009; 19(8): 1139-53. }\end{array}$ & Revisión sistemática cualitativa & $\begin{array}{l}215 \text { personas. } 12 \text { estudios in- } \\
\text { cluidos. Canadá (2), Israel (1), } \\
\text { Suecia (3), y EE.UU. (6). }\end{array}$ \\
\hline $\begin{array}{l}\text { (E16) Hwu YJ, Yu CC. Exploring } \\
\text { health behavior determinants for } \\
\text { people with chronic illness using } \\
\text { the constructs of planned behavior } \\
\text { theory. J Nurs Res. 2006; 14(4): } \\
261-70 .\end{array}$ & Estudio descriptivo & 36 personas. Taiwán \\
\hline $\begin{array}{l}\text { (E17) Chen KH, Chen ML, Lee S, } \\
\text { Cho HY, Weng LC. Self-manage- } \\
\text { ment behaviours for patients with } \\
\text { chronic obstructive pulmonary } \\
\text { disease: a qualitative study. J Adv } \\
\text { Nurs. 2008; 64(6): 595-604. }\end{array}$ & Estudio descriptivo & 18 personas. Taiwán \\
\hline $\begin{array}{l}\text { (E18) Hughes N, Closs SJ, Clark D. } \\
\text { Experiencing cancer in old age: a } \\
\text { qualitative systematic review. Qual } \\
\text { Health Res. 2009; 19(8): 1139-53. }\end{array}$ & $\begin{array}{l}\text { Estudio descriptivo interpre- } \\
\text { tativo }\end{array}$ & $\begin{array}{l}9 \text { personas. Aotearoa, (Welling- } \\
\text { ton) Nueva Zelanda }\end{array}$ \\
\hline $\begin{array}{l}\text { (E19) Freydberg N, Strain L, Tsu- } \\
\text { yuki RT, McAlister FA, Clark AM. } \\
\text { "If he gives in, he will be gone...": } \\
\text { the influence of work and place on } \\
\text { experiences, reactions and self-care } \\
\text { of heart failure in rural Canada. Soc } \\
\text { Sci Med. 2010; 70(7): 1077-83. }\end{array}$ & $\begin{array}{l}\text { Investigación cualitativa. Realis- } \\
\text { mo crítico }\end{array}$ & $\begin{array}{l}42 \text { personas. Edmonton, (Al- } \\
\text { berta) Canada }\end{array}$ \\
\hline
\end{tabular}




\section{Cultura de las Cuidados}

\begin{tabular}{|c|c|c|}
\hline $\begin{array}{l}\text { (E20) Williams AF, Manias E, } \\
\text { Walker R. The role of irrational } \\
\text { thought in medicine adherence: } \\
\text { people with diabetic kidney disease. } \\
\text { J Adv Nurs 2009; 65(10): 2108-17. }\end{array}$ & Diseño exploratorio descriptivo & $\begin{array}{c}23 \text { personas. Melbourne, } \\
\text { Australia }\end{array}$ \\
\hline $\begin{array}{l}\text { (E21) Davis R. Voices of Native } \\
\text { Hawaiian kupuna (elders) living } \\
\text { with chronic illness: "knowing who } \\
\text { I am". J Transcult Nurs. 2010; 21(3): } \\
\text { 237-45. }\end{array}$ & $\begin{array}{l}\text { Un diseño de investigación cua- } \\
\text { litativa, con etnoenfermería }\end{array}$ & 15 personas. Hawai, EE.UU \\
\hline $\begin{array}{l}\text { (E22) Mercado-Martínez FJ, } \\
\text { Hernández-Ibarra E. Las enferme- } \\
\text { dades crónicas desde la mirada de } \\
\text { los enfermos y los profesionales de } \\
\text { la salud: un estudio cualitativo en } \\
\text { México. Cad Saúde Pública 2007; } \\
\text { 23(9): 2178-2186. }\end{array}$ & $\begin{array}{c}\text { Una investigación cualitativa, } \\
\text { multicéntrica }\end{array}$ & $\begin{array}{c}67 \text { personas. Guadalajara, } \\
\text { San Luis Potosí y México DF. } \\
\text { México }\end{array}$ \\
\hline
\end{tabular}

Fuente: Elaboración propia 